\title{
Optimum System Geometry for S-band Multistatic Radar
}

\author{
A.Kh. Aboul-Seoud*, H. Taha ${ }^{\dagger}$, and A.E.S. Hafez ${ }^{\S}$
}

\begin{abstract}
Radar systems, based on Multistatic radar concept attracted a substantial attention in the recent years. The paper proposes system geometry for S-band Multistatic radar. This technique is used for detecting and tracking the small cross section area and stealthy aircrafts. The proposed geometrical structures are studied with different radars spacing to extend the detection coverage over the Monostatic radar used for air surveillance. The radar detection coverage is also studied with all possible stealthy aircraft paths to find the improvement achieved from using this type of radar. The simulation is done using Matlab program. The results show that the first system geometry with two transmitters and four receivers extends the detection coverage $80 \mathrm{Km}$ for small aircraft and $62 \mathrm{Km}$ for stealthy targets. The second system geometry with two transmitters and six receivers extends the detection coverage 85 $\mathrm{Km}$ for small targets and $69 \mathrm{Km}$ for stealthy targets. The achieved SNR from these system geometries guarantee a high probability of detection for small and stealthy aircraft detection.
\end{abstract}

Keywords: Multistatic radar, Stealthy Aircraft Detection

\section{1- Introduction}

Multistatic radar uses antennas at different locations for transmission and reception. This means that the transmitter and receiver are not co-located in the same place. There are different versions of Multistatic radar system does not specify how far the transmitting and receiving sites must be separated. In recent years, an extended growth of activities in the area of radar systems, based on the concept of the Multistatic radar is reported from many research centers and universities. Marc Brooker [1] presents the design and implementation of a signal level simulator supporting a wide variety of radar systems, focusing on Multistatic and netted radars. The simulator places few limits on the simulated system, and supports systems with arbitrary numbers of receivers, transmitters, and scatterers. Bezousek et. al. [2] described the Multistatic arrangement system with non-cooperative transmitters and various aspects of signal processing and signal parameters. Benson [3] investigates modeling method to optimize the location of receivers in order to achieve maximum coverage of aircraft moving around Cape Town International Airport. Several researches deal with improving the detection coverage using Multistatic radar systems [4-6]. These researches didn't study the radar coverage with different system geometry and various radar spacing. The paper proposes system geometry for S-band Multistatic radar for detecting and tracking the small cross section area and stealthy aircrafts. The proposed geometrical structures are studied with different radars spacing to extend the detection coverage over the Monostatic radar used for air surveillance. The radar detection coverage is also studied with all possible stealthy aircraft paths to find the improvement achieved from using this type of radar. The first section of the

\footnotetext{
* Professor, Faculty of Engineering, Alexandria University, Email: Ahmed_Khairy@yahoo.com .

$\dagger$ M.sc. student, Faculty of Engineering, Alexandria University, Email: Hazem Taha@yahoo.com .

$\S$ Affiliate Instructor, Faculty of Engineering, Alexandria University, Email: Alaahafez@iee.org .
} 
paper introduces theoretical description of the Multistatic radar. The second section presents the simulation results and the findings from this paper. The last section displays the conclusion of the paper.

\section{2-Theoretical Background}

A Multistatic radar system contains multiple spatially diverse Monostatic radar or bistatic radar components with a shared area of coverage. An important distinction system based on these individual radar geometries is the added requirement for some level of data fusion to take place between component parts. The spatial diversity afforded by Multistatic systems allows for different aspects of a target to be viewed simultaneously. The potential for information gain can give rise to a number of advantages over conventional systems. Multistatic radar is often referred to as 'Multisite' or 'netted' radar. Fig. 1. shows the idea of using all available sources of transmission for target localization and co-located receiver module, [7]. An obvious advantage of separating the receiver and the transmitter is that the receiver is passive and difficult to locate and which doesn't make it a target for e.g. Anti Radiation Missiles (ARM). Personnel are safe from ARM when located at the Rx. Separation also has effect on the effectiveness of Electronic Counter Measures (ECM) since the Tx and $\mathrm{Rx}$ are not co-located and therefore the Rx may be outside the main-lobe of the jammer or even outside the Line Of Sight (LOS). Since the receiver is not readily discovered it is suitable for covert operations. Several receivers can operate without disclose of their positions with the transmitter stand off by a large distance. Relations of Signal to Noise ratio $(\mathrm{S} / \mathrm{N})$ will be discussed. The range equation for a bistatic radar is derived in a manner completely analogous to that for a Monostatic radar. With this analog, the bistatic radar maximum-range equation can be written as [8-9],

$$
\left(R_{T} R_{R}\right)_{\max }=\left(\frac{P_{T} G_{T} G_{R} \lambda^{2} \sigma_{t} F_{T}^{2} F_{R}^{2}}{(4 \pi)^{3} K T_{s} B_{n}(S / N)_{\min } L_{T} L_{R}}\right)^{\frac{1}{2}}
$$

where $R_{T}, R_{R}$ are the transmitter and reciever to target range respectively, $P_{T}$ is the transmitted power, $G_{T}, G_{R}$ are the transmit and receive antenna power gain respectively, $\lambda$ is the transmitted signal wavelength, $\sigma_{t}$ is the bistatic radar cross section, $F_{T}, F_{R}$ are the transmit and receive propagation factor respectively, $K$ is the Boltzmann constant, $T_{\mathrm{s}}$ is the receive system noise temperature, $B_{n}$ is the noise bandwidth of the receiver, $S / N$ is the signal to noise ratio and $\mathrm{L}_{\mathrm{T}}, \mathrm{L}_{\mathrm{R}}$, are the transmit and receive system losses. The Multistatic from the radar equation is developed to evaluate Multistatic radar sensitivity properties. A fully coherent radar network is considered, which means that the radars comprising the whole network have a common and highly precise knowledge of time and space. The whole radar network is composed of $\mathrm{m}$ transmitters and $\mathrm{n}$ receivers. It is assumed that the whole network is well synchronized and works cooperatively such that each receiver is capable of receiving echoes due to any transmitters in the network. Under these assumptions, it is reasonable to calculate the overall radar sensitivity by summing up the partial signal to noise ratio, which is given by, [10].

$$
(S / N)_{\text {netted }}=\sum_{i=1}^{m} \sum_{j=1}^{n} \frac{P_{T i} G_{T i} G_{R j} \lambda_{i}^{2} \sigma_{t i j} F_{T i}^{2} F_{R j}^{2}}{(4 \pi)^{3} K T_{s} B_{n i} R_{T i}^{2} R_{R j}^{2} L_{T i} L_{R j}}
$$

Considering the simplest case where the radar parameters for every transmitter-receiver combination are the same, the Multistatic radar equation can be simplified as, 


$$
(S / N)_{\text {netted }}=\frac{P_{T} G_{T} G_{R} \lambda^{2} \sigma_{t} F_{T}^{2} F_{R}^{2}}{(4 \pi)^{3} K T_{s} B_{n} L_{T} L_{R}} \sum_{i=1}^{m} \sum_{j=1}^{n} \frac{1}{R_{T i}^{2} R_{R j}^{2}}
$$

From this equation it is clear to see that the Multistatic radar geometry, i.e. the positions of target and radar in the network, will have great influence on the overall Multistatic radar sensitivity.

\section{3- The Proposed System Geometries}

The proposed system geometries are demonstrated in Fig. 2. The first system geometry G1 consists of two transmitters and four receivers. The second system geometry G2 consists of two transmitters and six receivers. The spacing between radar stations is constant for the system geometry. The coordinate system must be converted for each radar station according to the following equations,

\begin{tabular}{l|l}
$R_{y r e f}=R_{\text {ref }} \cos \left(\theta_{\text {ref }}\right)$ & $R_{y 5}=R_{y r e f}+a$ \\
\hline$R_{x r e f}=R_{\text {ref }} \sin \left(\theta_{r e f}\right)$ & $R_{x 5}=R_{x r e f}+a$ \\
\hline$R_{y 2}=R_{y r e f}$ & $R_{y 6}=R_{y r e f}+a$ \\
\hline$R_{x 2}=R_{x r e f}-a$ & $R_{x 6}=R_{x r e f}-a$ \\
\hline$R_{y 3}=R_{y r e f}+2 a$ & $R_{y x 1}=R_{y r e f}+a$ \\
\hline$R_{x 3}=R_{x r e f}$ & $R_{x x 1}=R_{x r e f}$ \\
\hline$R_{y 4}=R_{y r e f}+2 a$ & $R_{y x 2}=R_{y r e f}+a$ \\
\hline$R_{x 4}=R_{x r e f}-a$ & $R_{x x 2}=R_{x r e f}-a$
\end{tabular}

\section{4- Simulation Results}

The system geometries are simulated under Matlab program environment to obtain the radar sensitivity under the proposed conditions, and demonstrate all possible radar spacing to achieve an optimum radar arrangement satisfying the optimum detection. The radar transmitter parameters are illustrated in table 1. Multistatic radar system geometry G1 and G2 are simulated with radar spacing from $5 \mathrm{Km}$ to $70 \mathrm{Km}$ for aircraft range from $50 \mathrm{Km}$ to 140 $\mathrm{Km}$. It is found that the optimum radar spacing for the system geometry G1 is $48 \mathrm{Km}$ and 50 $\mathrm{Km}$ for $\mathrm{G} 2$. Fig. 3. shows the received $\mathrm{S} / \mathrm{N}$ at different radar spacing and target ranges for system geometry G1with small aircrafts $(\sigma=0.1 \mathrm{~m} 2)$, and stealthy aircrafts $(\sigma=0.025 \mathrm{~m} 2)$. A comparison between Multistatic radar system geometry G1 and Monostatic radar with small cross section is investigated in Fig. 4. Same comparative study is demonstrated in Fig. 5. for stealthy aircrafts. Fig. 6. shows the received $\mathrm{S} / \mathrm{N}$ at different radar spacing and target ranges for system geometry G2 with small aircrafts $(\sigma=0.1 \mathrm{~m} 2)$, and stealthy aircrafts $(\sigma=0.025$ $\mathrm{m} 2$ ). Received S/N at different azimuth angles and target ranges for system geometry G2 is demonstrated in Fig. 7. The results are concluded in Fig. 8. The system geometry G1 extends the detection coverage $80 \mathrm{Km}$ for small aircraft and $62 \mathrm{Km}$ for a stealthy target over the Monostatic radar. The second system geometry G2 improves the detection coverage for the small aircrafts by $85 \mathrm{Km}$ and $69 \mathrm{Km}$ for a stealthy aircrafts. 
Table 1 Radar transmitter parameters

\begin{tabular}{c|c||c|c}
\hline \hline Parameter & Value & Parameter & Value \\
\hline \hline $\mathrm{P}_{\mathrm{T}}(\mathrm{Kwatt})$ & 200 & $\mathrm{~B}_{\mathrm{n}}(\mathrm{MHz})$ & 1 \\
\hline $\mathrm{G}_{\mathrm{T}}, \mathrm{G}_{\mathrm{R}}(\mathrm{dB})$ & 33 & $\mathrm{~F}_{\mathrm{T},} \mathrm{F}_{\mathrm{R}}$ & 1 \\
\hline $\mathrm{F}(\mathrm{MHz})$ & 2450 & $\mathrm{~L}_{\mathrm{T}}, \mathrm{L}_{\mathrm{R}}(\mathrm{dB})$ & 5 \\
\hline
\end{tabular}

\section{5- Conclusion}

The paper proposes two Multistatic radar system geometries for S-band radar to improve the detection coverage of the small and stealthy aircrafts. The first system geometry G1 consists of 4 transmitters and two receivers while the second one consists of six transmitters and two receivers. The optimum radar spacing for G1 is $48 \mathrm{Km}$ and $50 \mathrm{Km}$ for system geometry G2. The simulation results show that system geometry G1 detection coverage extended $80 \mathrm{Km}$ for small aircrafts over detected by Monostatic radar and $62 \mathrm{Km}$ for stealthy aircrafts. System geometry G2 extends the detection coverage $85 \mathrm{Km}$ with small aircrafts and $69 \mathrm{Km}$ for stealthy aircrafts. The results show that the extended range from the first system geometry satisfies the need for the small and stealthy aircrafts detection with economic number of radar stations.

\section{6- References}

[1] Marc Brooker, "The Design and Implementation of a Simulator for Multistatic Radar Systems,"PHD Thesis, University of Cape Town, June, 2008.

[2] Pavel Bezousek, Vladimir Schejbal," Bistatic and Multistatic Radar Systems," Radioengineering, Vol. 17, No. 3, Sep. 2008.

[3] Benson Chan, " Receiver Site Optimisation for Passive Coherent Location (PCL) Radar System,"Graduation Project, University of Cape Town, Oct., 2008.

[4] Vladimír S., Dušan Č., Zdeněk N., Jan P., Jiří K., Pavel B., Ondřej F.," Multipath Propagation of UWB Through-Wall Radar and EMC Phenomena," Radioengineering, Vol. 15, No. 4, Dec. 2006.

[5] Pavel Bezoušek, Marek Pola, Jan Pidanič," Detection of Multiple Targets by a Multistatic Radar," Number 4., Volume IV., Dec. 2009.

[6] Steven Kay," Waveform Design for Multistatic Radar Detection," Dept. of Electrical, Computer, and Biomedical Engineering, University of Rhode Island Kingston, RI 02881, 2007.

[7] Terje Johnsen and Karl Erik Olsen," Bi- and Multistatic Radar," Advanced Radar Signal and Data Processing Educational Notes RTO-EN-SET-086, Paper 4. Neuilly-sur-Seine, France: RTO. Available from: http://www.rto.nato.int/abstracts.asp, visited Oct. 2010

[8] Merril I. Skolnik," Radar Handbook, Second Edition," McGraw-Hill, 1990

[9] Bassem R. Mahafza," Radar Systems Analysis and Design Using MATLAB," CRC Press, USA, 2000

[10] Chris Baker, "Multistatic radar processing and systems," Advanced Radar Signal and Data Processing Educational Notes RTO-EN-SET-133, Paper 4. Neuilly-sur-Seine, France: RTO. Available from: http://www.rto.nato.int/abstracts.asp, visited Oct. 2010 


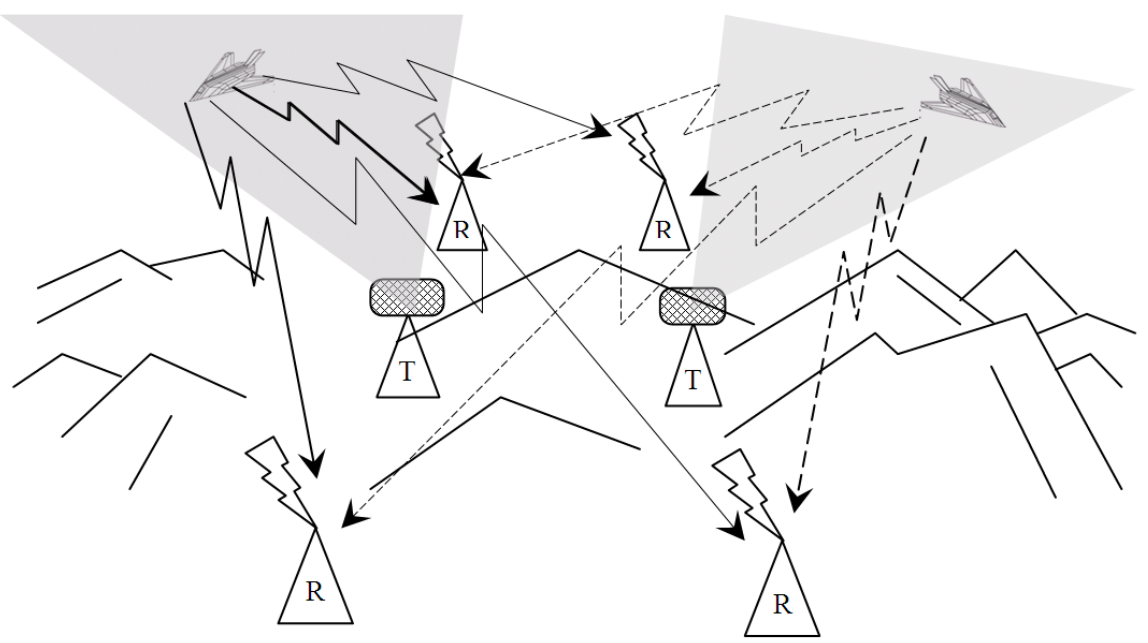

Fig. 1 The combined use of available sources in the process of producing the air picture

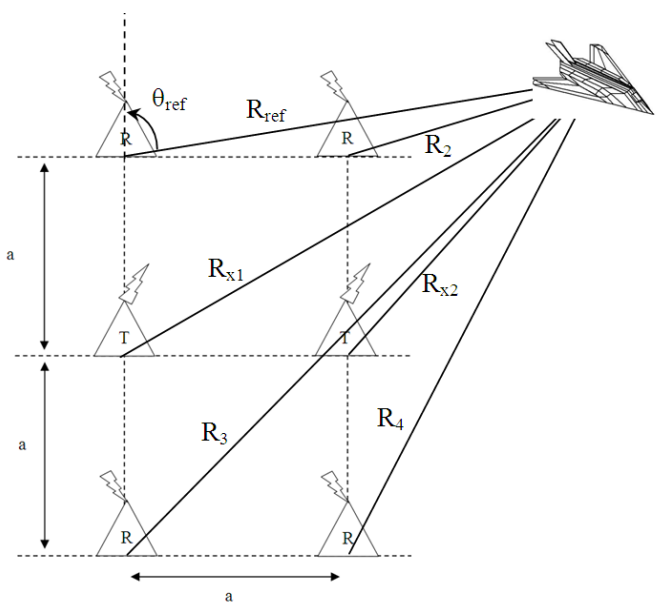

(a)

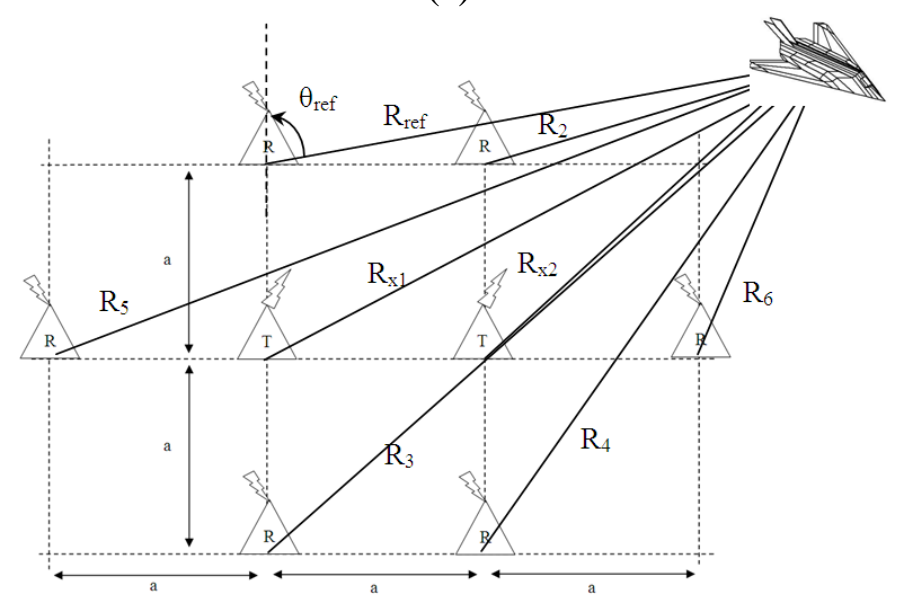

(b)

Fig. 2 Multistatic Radar System Geometry (a) System Geometry G1 and (b) System Geometry G2 


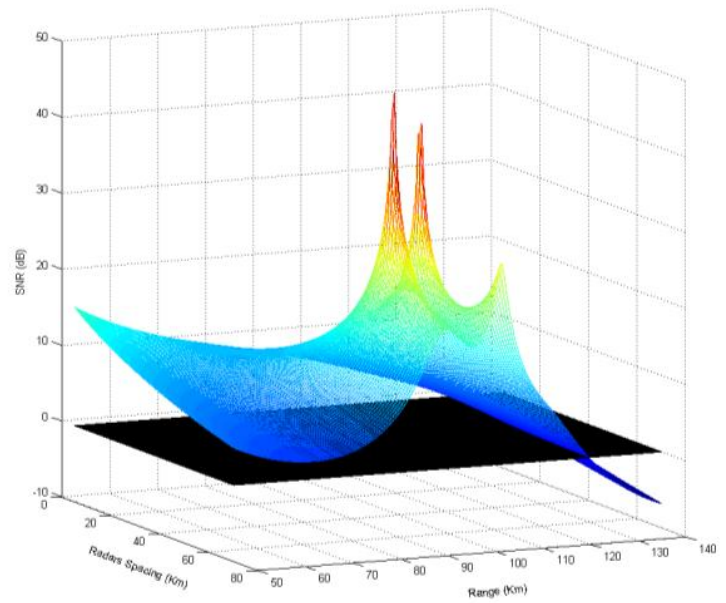

(a)

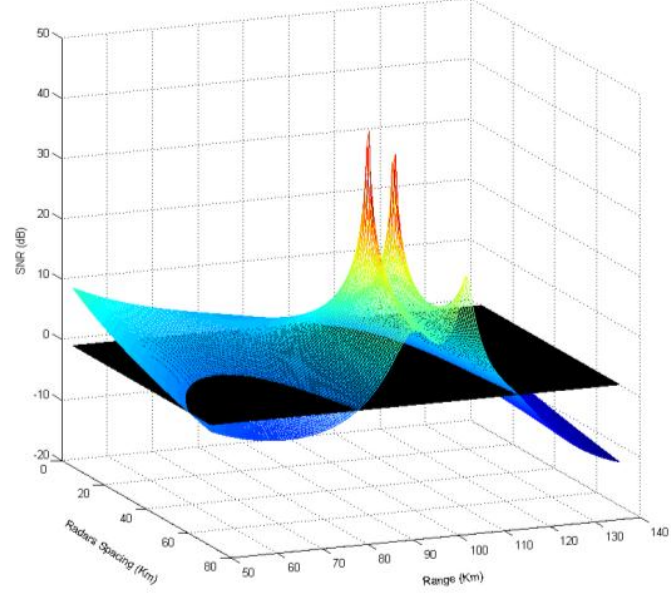

(b)

Fig. 3 Received $\mathrm{S} / \mathrm{N}$ at different radar spacing and target ranges for system geometry G1 (a) Small Aircrafts $\sigma=0.1 \mathrm{~m}^{2}$, (b) Stealthy Aircrafts $\sigma=0.025 \mathrm{~m}^{2}$.

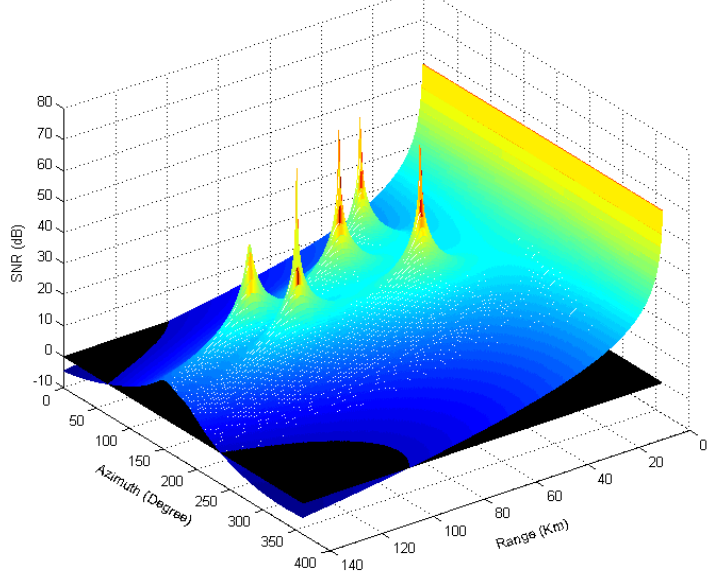

(a)

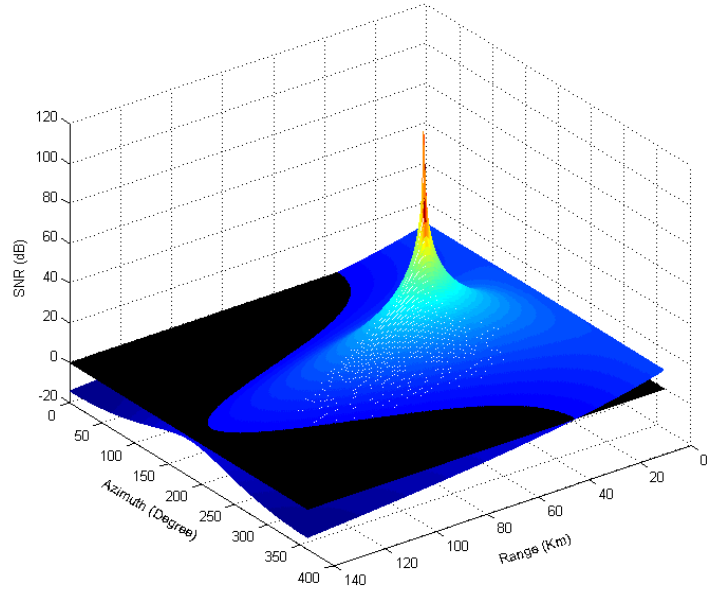

(b)

Fig. 4 Received $\mathrm{S} / \mathrm{N}$ at different azimuth angels and target ranges with small aircrafts $\sigma=0.1 \mathrm{~m}^{2}$ (a) Multistatic radar G1, (b) Monostatic radar

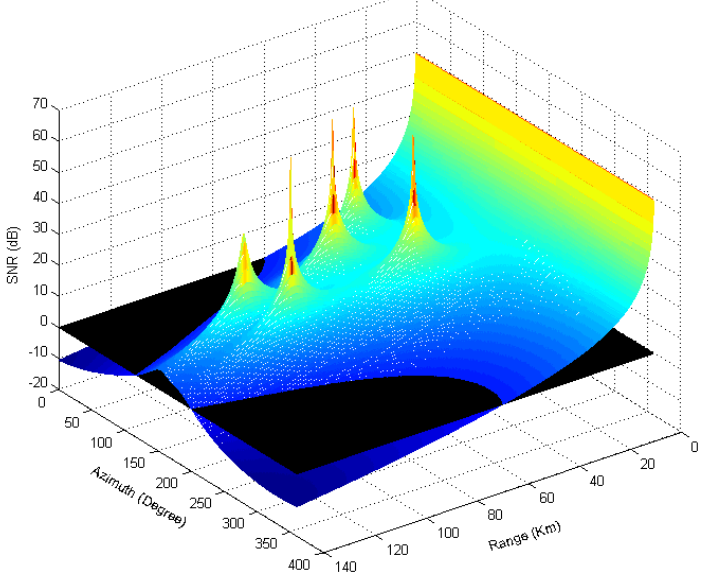

(a)

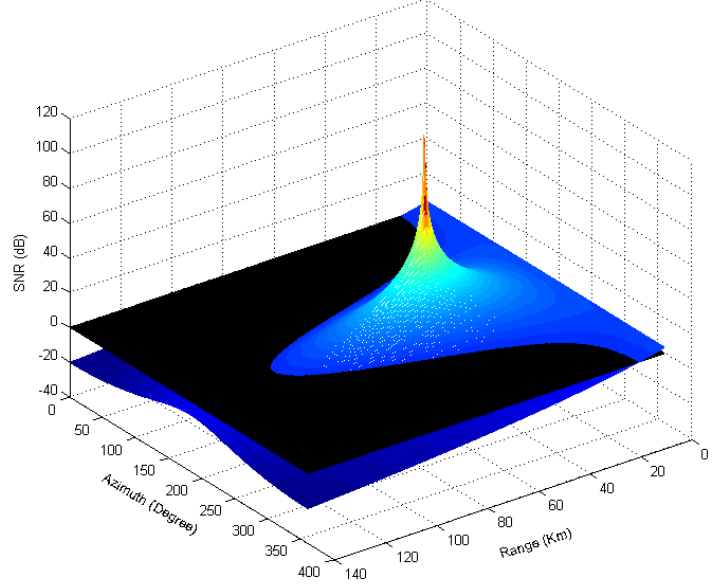

(b)

Fig. 5 Received $\mathrm{S} / \mathrm{N}$ at different azimuth angels and target ranges with stealthy aircrafts $\sigma=0.025 \mathrm{~m}^{2}$ (a) Multistatic radar G1, (b) Monostatic radar 


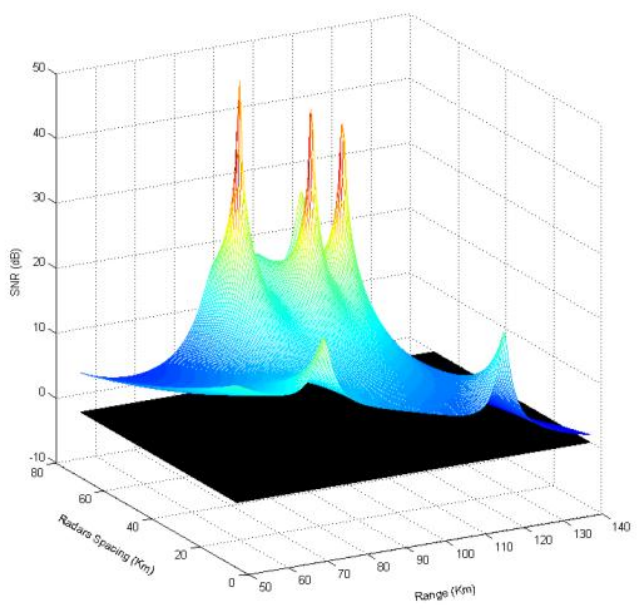

(a)

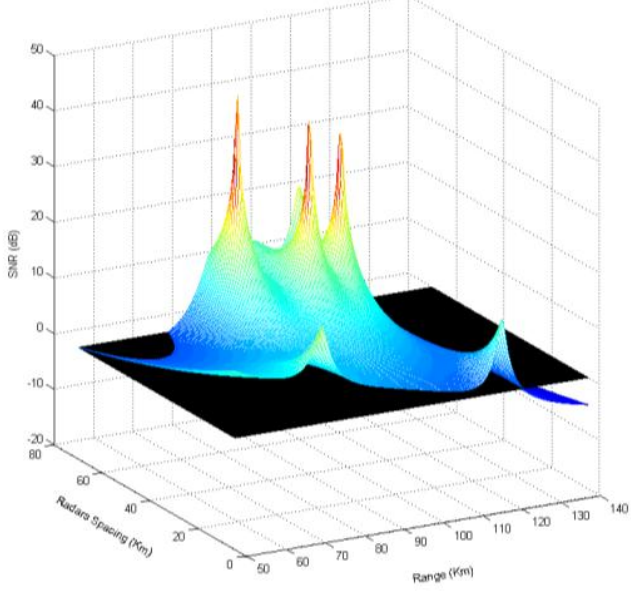

(b)

Fig. 6 Received S/N at different radar spacing and target range for system geometry G2 (a) Small Aircrafts $\sigma=0.1 \mathrm{~m}^{2}$, (b) Stealthy Aircrafts $\sigma=0.025 \mathrm{~m}^{2}$.

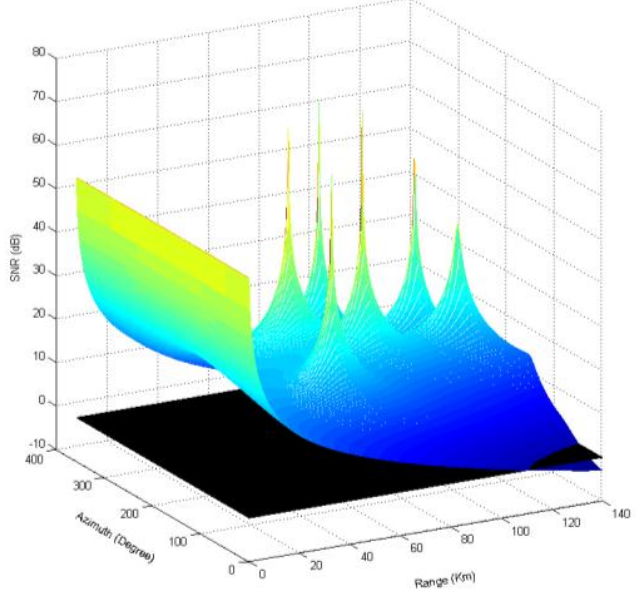

(a)

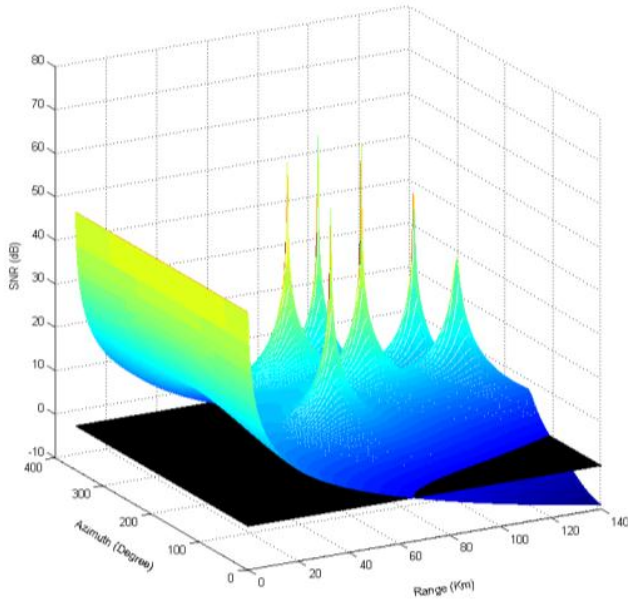

(b)

Fig. 7 Received S/N at different azimuth angels and target ranges G2 (a) Small Aircrafts $\sigma=0.1 \mathrm{~m}^{2}$, (b) Stealthy Aircrafts $\sigma=0.025 \mathrm{~m}^{2}$.

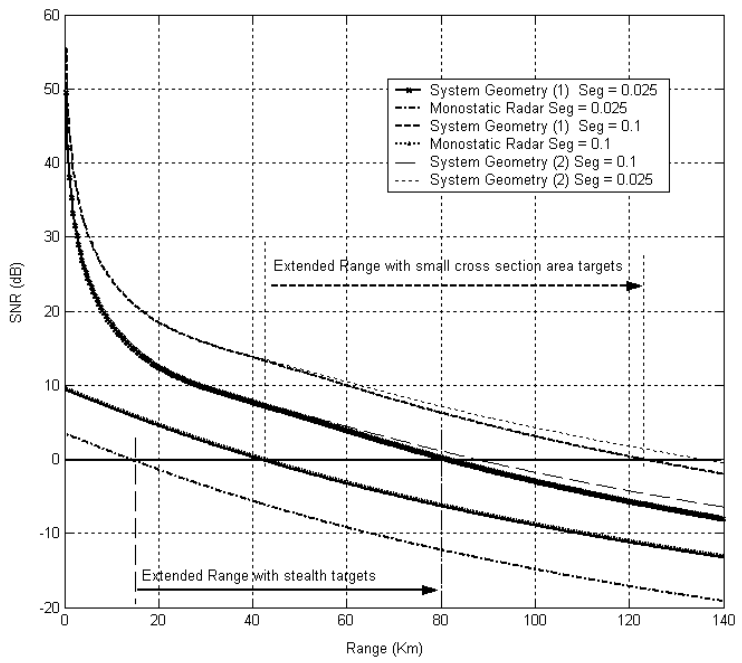

Fig. 8 Received $\mathrm{S} / \mathrm{N}$ versus target range for Multistatic radar G1, G2, and Monostatic radar with target cross section $\sigma=0.1 \mathrm{~m}^{2}$ and $\sigma=0.025 \mathrm{~m}$ 